\title{
Safety of As-Needed Budesonide-Formoterol in Mild Asthma: Data from the Two Phase III SYGMA Studies
}

\author{
J. Mark FitzGerald ${ }^{1} \cdot$ Paul M. O'Byrne ${ }^{2}$ Eric D. Bateman ${ }^{3} \cdot$ Peter J. Barnes $^{4} \cdot$ Jinping Zheng $^{5} \cdot$ Stefan Ivanov $^{6}$. \\ Rosa Lamarca ${ }^{7}$. Ulrika Larsdotter ${ }^{6}$. Ulrika Emerath ${ }^{6} \cdot$ Gerreke Jansen $^{6} \cdot$ Margareta Puu $^{6} \cdot$ Vijay K. T. Alagappan ${ }^{8}$. \\ Filip Surmont ${ }^{9} \cdot$ Helen K. Reddel $^{10}$
}

Accepted: 27 December 2020 / Published online: 6 February 2021

(c) The Author(s) 2021

\begin{abstract}
Introduction Budesonide-formoterol taken as needed is an emerging treatment for mild asthma.

Objective We used data from the SYGMA studies to assess the safety of As-needed budesonide-formoterol compared with As-needed terbutaline and compared with maintenance budesonide.

Methods SYGMA 1 and 2 were 52-week, double-blind, parallel-group studies in patients aged $\geq 12$ years with physicianassessed mild asthma. Patients were randomized to As-needed budesonide-formoterol 200/6 $\mu \mathrm{g}$, twice-daily budesonide $200 \mu \mathrm{g}$ as maintenance plus As-needed terbutaline $0.5 \mathrm{mg}$, and As-needed terbutaline $0.5 \mathrm{mg}$ (SYGMA 1 only). Adverse events (AEs), serious AEs (SAEs), discontinuations due to AEs (DAEs), and study-defined asthma-related discontinuations from corresponding treatment groups in both studies were pooled. SYGMA 1 data were used for comparisons with Asneeded terbutaline alone.

Results The pooled analysis included 3366 patients in the As-needed budesonide-formoterol group and 3369 in the budesonide maintenance group, with AEs in $40.8 \%$ and $42.5 \%$ of patients, respectively. Common AEs included viral upper respiratory tract infection (viral URTI) and URTI. SAE, DAE, and asthma-related discontinuation rates were similar with As-needed budesonide-formoterol and maintenance budesonide. Potential local and systemic corticosteroid class effects were reported in $\leq 1 \%$ of patients for each budesonide-containing regimen. In SYGMA 1 , AEs were more common in the As-needed terbutaline $(n=1277)$ than As-needed budesonide-formoterol $(n=1277)$ groups $(42.7$ vs. $38.0 \%)$, as were DAEs ( 2.9 vs. $0.8 \%$ ) and asthma-related discontinuations (1.6 vs. $0.3 \%$ ).

Conclusions Budesonide-formoterol anti-inflammatory reliever therapy is generally well-tolerated in patients with mild asthma and has a safety profile similar to that of daily budesonide. No new safety signals were identified.
\end{abstract}

ClinicalTrial.gov Identifiers NCT02149199 (SYGMA 1) and NCT02224157 (SYGMA 2).

J. Mark FitzGerald

Mark.fitzgerald@vch.ca

Extended author information available on the last page of the article 


\section{Key Points}

As-needed budesonide-formoterol was well-tolerated in patients with mild asthma, and no new safety signals were identified.

As-needed budesonide-formoterol had a safety profile similar to that of twice-daily maintenance budesonide but with a lower total exposure to corticosteroid. The safety profile of as-needed budesonide-formoterol was favorable compared with as-needed terbutaline.

Only a very small proportion of patients receiving asneeded budesonide-formoterol required sustained high use of their treatment.

\section{Introduction}

The combination of an inhaled corticosteroid and long-acting $\beta_{2}$-agonist (ICS-LABA) inhaler therapy is the mainstay of treatment for moderate and severe asthma $[1,2]$. Currently, budesonide-formoterol (Symbicort ${ }^{\circledR}$ Turbuhaler $^{\circledR}$ ), a combination ICS-LABA, is approved for use as daily maintenance therapy in more than 100 countries worldwide. In many of these, it is also approved as both maintenance and reliever therapy (MART), a strategy associated with clinical outcomes superior to those with conventional approaches in patients with persistent asthma, particularly those with a history of severe exacerbations [3].

The safety profile of budesonide-formoterol in patients with moderate-severe asthma is well-established from numerous clinical studies, both for maintenance-only therapy and for MART [4-13]. Adverse events (AEs) reported to be common $(\geq 1$ to $<10 \%)$ with budesonide-formoterol treatment include candida infections in the oropharynx, headache, tremor, palpitations, throat irritation, coughing, and hoarseness [14]. Some of these are pharmacologically predictable, being related to the class and dose of drug (ICS or $\beta_{2}$-adrenoceptor agonist), and others to the mode of administration, such as drug deposition in the oropharynx through the use of inhalers $[14,15]$. The majority of these AEs are mild, and clinically significant AEs are usually associated only with high doses of ICS, which are recommended for only a minority of patients [1].

Evidence for the efficacy of budesonide-formoterol as anti-inflammatory reliever therapy alone (i.e., without maintenance ICS-containing treatment) in mild asthma recently became available from two 52-week, double-blind phase III
SYGMA (Symbicort ${ }^{\circledR}$ Given as Needed in Mild Asthma) studies $[5,7]$.

On the basis of evidence from these studies, and supported by two additional open-label 12-month studies [16, 17], the Global Initiative for Asthma (GINA) strategy now recommends As-needed low-dose combination ICS-formoterol as a preferred treatment for mild asthma in adults and adolescents aged $\geq 12$ years (step 1 and 2 treatment) $[1,18]$. Daily low-dose ICS plus As-needed short-acting $\beta_{2}$-agonist (SABA) remains an alternative preferred step 2 controller option in adult and adolescent patients and the preferred step 2 controller for children with asthma [1].

SABA-only treatment (without ICS) is no longer recommended by GINA for adults and adolescents for safety reasons $[1,18]$ : SABAs do not address the airway inflammation seen in mild asthma, and regular use or over-use of SABA is associated with various adverse outcomes as well as an increased risk of severe exacerbations, with potentially fatal consequences [18-20], and an increased risk of AEs from courses of systemic corticosteroids used to treat severe exacerbations [21, 22]. However, patients with mild asthma often believe they do not need any treatment other than SABA [23, 24], and patients and practitioners may be concerned about using an ICS-containing product for symptom relief, given the lingering widespread aversion to the use of steroids in many countries.

Therefore, this analysis examined the safety of budesonide-formoterol as anti-inflammatory reliever therapy compared with As-needed SABA and compared with daily ICS in patients with mild asthma enrolled in the two SYGMA studies $[5,7]$.

\section{Methods}

\subsection{Study Design and Participants}

SYGMA 1 (NCT022149199) [7] and SYGMA 2 (NCT02224157) [5] were 52-week, double-blind, randomized, multinational, parallel-group studies. Detailed descriptions of the study design, patient inclusion/exclusion criteria, and study outcomes are available in the original publications [5, 7].

Briefly, both studies included patients aged $\geq 12$ years with physician-assessed mild asthma, and for whom GINA (2012) step 2 treatment (regular low-dose ICS or leukotriene receptor antagonist [LTRA]) was indicated. There were two pre-specified patient subgroups on the basis of their prior treatment: those with asthma uncontrolled on as-needed SABA (subgroup 1) and those with asthma controlled on low-dose ICS or LTRA plus As-needed SABA (subgroup 2).

In SYGMA 1, patients were randomized to twice-daily placebo plus As-needed terbutaline $0.5 \mathrm{mg}$ (Bricanyl ${ }^{\circledR}$ 
Turbuhaler ${ }^{\circledR}$ [AstraZeneca, Sweden]; corresponding to a delivered dose of $0.4 \mathrm{mg}$ ); twice-daily placebo plus Asneeded budesonide-formoterol 200/6 $\mu \mathrm{g}$ (Symbicort ${ }^{\circledR}$ Turbuhaler ${ }^{\circledR}$ [AstraZeneca, Sweden]; corresponding to a delivered dose of 160/4.5 $\mu \mathrm{g}$ ); or twice-daily budesonide $200 \mu \mathrm{g}$ (Pulmicort ${ }^{\circledR}$ Turbuhaler ${ }^{\circledR}$ [AstraZeneca, Sweden]) as maintenance, plus As-needed terbutaline $0.5 \mathrm{mg}$ (Bricanyl ${ }^{\circledR}$ Turbuhaler $^{\circledR}$ [AstraZeneca, Sweden]) (herein referred to as budesonide maintenance). In SYGMA 2, patients were randomized to placebo plus As-needed budesonide-formoterol (as for SYGMA 1) or to budesonide maintenance (as for SYGMA 1).

\subsection{Adverse Event Reporting}

Detailed descriptions of the methodology of AE monitoring and reporting are provided in the individual study protocols and statistical analysis plans for SYGMA 1 [7] and 2 [5], which are available online at the site of their publication. Briefly, AE data were collected from the start of run-in (visit 2) and serious AEs (SAEs) from the time of informed consent (visit 1), at every clinical visit, and through to the last telephone follow-up or last contact. AEs were assigned a system organ class and preferred term according to the Medical Dictionary for Regulatory Activities (MedDRA ${ }^{\circledR}$ ), version 20.0. An $\mathrm{AE}$ was considered serious if it met one or more of the following International Conference on Harmonisation criteria [25]: resulting in death, immediately life threatening, required inpatient hospitalization or prolongation of existing hospitalization, resulted in significant or persistent disability/incapacity, was a congenital abnormality or birth defect, or was an important medical event jeopardizing the patient or that required medical intervention to avoid any of these outcomes. Asthma symptoms (cough, wheeze, etc.) were recorded as AEs only when they met SAE or discontinuation due to AE (DAE) criteria, or the event was new to the patient or inconsistent with the pre-existing asthma history, as judged by the investigator. Criteria for an asthmarelated discontinuation were defined in the study as follows: a severe asthma exacerbation of 3 weeks' duration (in both studies) [5, 7]; two severe exacerbations during the same 3 -month period or a total of three severe exacerbations during the study in SYGMA 1 [7]; or three severe exacerbations within a 6-month period in SYGMA 2 [5].

Any fatal events were assessed for their relationship to asthma by an independent external adjudication committee and for their relationship to study treatment by the investigators.

\subsection{Data Analyses}

Following a pre-specified statistical analysis plan, data from corresponding treatment groups in SYGMA 1 and SYGMA
2 (As-needed budesonide-formoterol and budesonide maintenance treatment) were pooled to assess safety. As patients received As-needed terbutaline alone only in SYGMA 1 [7], safety comparisons for this treatment group were only made with As-needed budesonide-formoterol data from SYGMA 1 (i.e., non-pooled data). Corticosteroid exposure data were also pooled. In addition to the ICS component of the Asneeded combination and maintenance treatments, patients were permitted to receive additional systemic corticosteroids for the treatment of severe exacerbations in both studies [5, 7], and open-label inhaled budesonide for moderate exacerbations or prolonged poor asthma control in SYGMA 1 [7]. Such corticosteroid use was included in the exposure calculations.

Safety outcomes assessed were AEs, fatal and non-fatal SAEs, DAEs, and AEs representing potential corticosteroid class effects or $\beta_{2}$-agonist class effects (see the electronic supplementary material). Drug class effects were not systematically assessed.

AEs in patients with high use of As-needed budesonideformoterol, defined as $>8$ or $>12$ inhalations/day on at least 1 day, and in those with episodes of sustained high As-needed use, defined as $>8$ or $>12$ inhalations for either $\geq 7$ or $\geq 14$ consecutive days, were also included in safety analyses; in the budesonide maintenance group, 'high Asneeded' use refers to the use of terbutaline. The date and time of use of all randomized inhalers (maintenance and As-needed) was recorded electronically throughout SYGMA 1 and SYGMA 2.

In both studies, safety was evaluated in the safety analysis set (SAS), defined as all patients receiving any investigational product, classified according to the treatment they actually received $[5,7]$. The pooled SAS was a combination of the individual study SAS. A full analysis set (FAS), which was identical to the SAS in both SYGMA studies [5, 7], was used where safety parameters had been planned as part of the efficacy assessment in these studies and in the current report, which includes high As-needed use and corticosteroid exposure data. AEs while on randomized treatment (including the first day after stopping treatment), and patient deaths at any time during the study, were included in the assessment of the pooled safety population.

No formal statistical hypothesis testing was conducted. Descriptive statistics were used to summarize AE parameters by treatment group. Selected comparisons were made for subgroups of sex (male vs. female) and age ( $\geq 12$ to $<$ $18, \geq 18$ to $<50, \geq 50$ to $<65$, and $\geq 65$ years). 


\section{Results}

In SYGMA 1, there were 1277 evaluable patients in the As-needed budesonide-formoterol group, 1282 in the budesonide maintenance group, and 1277 in the As-needed terbutaline group (FAS and SAS) [7]. In SYGMA 2, there were 2089 evaluable patients in the As-needed budesonideformoterol group and 2087 in the budesonide maintenance group (FAS) [5]. The total number of patients in the pooled SAS was 6735, with 3366 in the As-needed budesonideformoterol group and 3369 in the budesonide maintenance group.

A similar proportion of patients in SYGMA 1 and SYGMA 2 were taking low-dose ICS for at least 30 days prior to enrolment (47.4 and $48.1 \%$, respectively). The respective proportions of patients taking LTRA over this period were 8.2 and $5.6 \%$.

\subsection{Demographics and Baseline Characteristics}

Patient baseline demographic and clinical characteristics were well balanced between As-needed terbutaline, Asneeded budesonide-formoterol, and budesonide maintenance groups, as previously reported for SYGMA 1 [7]. Treatment groups were likewise balanced in SYGMA 2 [5].
Baseline characteristics were similar between As-needed budesonide-formoterol and budesonide maintenance groups in the pooled safety population (Table 1). Overall, patients in the pooled safety population were considered representative of the target population of patients with mild asthma for whom use of low-dose ICS had been appropriate (based on clinical recommendations at the time the study protocol was finalized, i.e., GINA 2012 step 2).

\subsection{Treatment Exposure}

Duration of exposure to study treatment was slightly greater in the As-needed budesonide-formoterol group than in the As-needed terbutaline group in SYGMA 1 (343.5 \pm 71.7 vs. $329.8 \pm 91.1$ mean treatment days) and similar between treatments in SYGMA $2(349.3 \pm 62.1$ vs. $346.7 \pm 67.9$ mean treatment days). In SYGMA 1, 89.8\% of patients in the As-needed budesonide-formoterol group versus $85.0 \%$ of patients in the As-needed terbutaline group had received study treatment for $>48$ weeks, whereas $>90 \%$ of patients in both treatment groups received treatment for $>48$ weeks in SYGMA 2.

For the pooled safety population, duration of exposure to study treatment was similar in the As-needed budesonideformoterol and budesonide maintenance treatment groups ( $347.1 \pm 66.0$ vs. $344.5 \pm 70.8$ mean treatment days). Study

Table 1 Demographics and baseline characteristics

\begin{tabular}{|c|c|c|c|c|c|}
\hline \multirow[t]{2}{*}{ Characteristics } & \multicolumn{3}{|c|}{ SYGMA 1 and SYGMA 2 pooled safety population } & \multicolumn{2}{|l|}{ SYGMA 1} \\
\hline & $\begin{array}{l}\text { As-needed budesonide- } \\
\text { formoterol } 200 / 6 \mu \mathrm{g} \\
(n=3366)\end{array}$ & $\begin{array}{l}\text { Budesonide mainte- } \\
\text { nance } 200 \mu \mathrm{g} \text { bid } \\
(n=3369)\end{array}$ & $\begin{array}{l}\text { Total } \\
(n=6735)\end{array}$ & $\begin{array}{l}\text { As-needed budesonide- } \\
\text { formoterol } 200 / 6 \mu \mathrm{g} \\
(n=1277)\end{array}$ & $\begin{array}{l}\text { As-needed } \\
\text { terbutaline } 0.5 \\
\mathrm{mg} \\
(n=1277)\end{array}$ \\
\hline Age, years & $40.7 \pm 16.9$ & $40.1 \pm 17.0$ & $40.4 \pm 16.9$ & $39.8 \pm 16.9$ & $40.0 \pm 16.3$ \\
\hline Female sex & 2085 (61.9) & $2086(61.9)$ & $4171(61.9)$ & $777(60.8)$ & $771(60.4)$ \\
\hline \multicolumn{6}{|l|}{ Years since asthma diagnosis } \\
\hline Median & 7.3 & 6.9 & 7.1 & 6.5 & 6.3 \\
\hline Range & $0.4-65.7$ & $0.4-71.2$ & $0.4-71.2$ & $0.4-65.7$ & $0.5-62.4$ \\
\hline Pre-BD FEV $1, \%$ predicted $^{\mathrm{a}}$ & $84.3 \pm 14.0$ & $84.2 \pm 13.9$ & $84.2 \pm 14.0$ & $84.2 \pm 14.2$ & $84.1 \pm 14.1$ \\
\hline Reversibility, $\%^{\mathrm{b}}$ & $15.0 \pm 12.0$ & $15.0 \pm 12.5$ & $15.0 \pm 12.2$ & $14.9 \pm 11.3$ & $14.4 \pm 11.5$ \\
\hline $\begin{array}{l}\text { Severe exacerbation in previous } \\
12 \text { months }\end{array}$ & $716(21.3)$ & $701(20.8)$ & $1417(21.0)$ & $257(20.1)$ & $256(20.0)$ \\
\hline \multicolumn{6}{|l|}{ Pre-study treatment } \\
\hline Uncontrolled on BD & $1524(45.3)$ & $1551(46.0)$ & $3075(45.7)$ & $565(44.2)$ & $565(44.2)$ \\
\hline Controlled on ICS or LTRA & $1842(54.7)$ & $1818(54.0)$ & $3660(54.3)$ & $712(55.8)$ & $712(55.8)$ \\
\hline Controlled on ICS & $1626(48.3)$ & $1584(47.0)$ & $3210(47.7)$ & $611(47.8)$ & $619(48.5)$ \\
\hline
\end{tabular}

All data are $n(\%)$ or mean \pm standard deviation unless specified otherwise

$B D$ bronchodilator, bid twice daily, $F E V_{1}$ forced expiratory volume in 1 second, ICS inhaled corticosteroid, $L T R A$ leukotriene receptor antagonist ${ }^{a} \mathrm{FEV}_{1}$ assessment: $n=3356$ (As-needed budesonide-formoterol), $n=3357$ (budesonide maintenance) and $n=6713$ (total)

${ }^{\mathrm{b}}$ Reversibility assessment: $n=3340$ (As-needed budesonide-formoterol), $n=3335$ (budesonide maintenance), and $n=6675$ (total) 
treatment was received for $>48$ weeks in $\geq 90 \%$ of patients in both groups.

\subsection{Common Adverse Events}

AEs were more frequent in the As-needed terbutaline group than in the As-needed budesonide-formoterol group (545 [42.7\%] vs. 485 [38.0\%] patients) in SYGMA 1 [7]. A similar proportion of patients using As-needed budesonide-formoterol and budesonide maintenance treatment experienced an AE in the pooled safety population of SYGMA 1 and SYGMA 2 (1372 [40.8\%] vs. 1431 [42.5\%] patients) (Table 2). The most common AEs (occurring in $\geq 2 \%$ of patients) are summarized in Table 3 . The three most commonly reported AEs in SYGMA 1 were viral upper respiratory tract infection (viral URTI), URTI, and asthma in both the As-needed terbutaline and As-needed budesonide-formoterol groups [7]. Viral URTI and URTI occurred in a similar proportion of patients in the As-needed terbutaline and As-needed budesonide-formoterol groups, whereas asthma was reported as an $\mathrm{AE}$ in more patients in the As-needed terbutaline group (109 [8.5\%] vs. 37 [2.9\%] patients in the budesonide-formoterol group). The pooled analysis also showed similarity in the nature and incidence of the three most common AEs between the As-needed budesonide-formoterol and budesonide maintenance groups: URTI, viral URTI, and asthma. The safety profile of Asneeded budesonide-formoterol compared with As-needed terbutaline and with budesonide maintenance treatment did

Table 2 Summary of adverse events (safety analysis set)

\begin{tabular}{|c|c|c|c|c|c|}
\hline \multirow[t]{2}{*}{$\mathrm{AE}$} & \multicolumn{3}{|c|}{ SYGMA $1+$ SYGMA 2 pooled safety population } & \multicolumn{2}{|l|}{ SYGMA 1} \\
\hline & $\begin{array}{l}\text { As-needed budesonide- } \\
\text { formoterol } 200 / 6 \mu \mathrm{g} \\
(n=3366)\end{array}$ & $\begin{array}{l}\text { Budesonide maintenance } \\
200 \mu \mathrm{g} \text { bid } \\
(n=3369)\end{array}$ & $\begin{array}{l}\text { Total } \\
(n=6735)\end{array}$ & $\begin{array}{l}\text { As-needed budesonide- } \\
\text { formoterol } 200 / 6 \mu \mathrm{g} \\
(n=1277)\end{array}$ & $\begin{array}{l}\text { As-needed } \\
\text { terbutaline } 0.5 \\
\mathrm{mg} \\
(n=1277)\end{array}$ \\
\hline Any AE & $1372(40.8)$ & $1431(42.5)$ & 2803 (41.6) & $485(38.0)$ & $545(42.7)$ \\
\hline $\begin{array}{l}\text { Any AE with } \\
\text { an outcome of } \\
\text { death }\end{array}$ & $1(<0.1)$ & $3(<0.1)$ & $4(<0.1)$ & 0 & 0 \\
\hline Any SAE & $104(3.1)$ & $110(3.3)$ & $214(3.2)$ & $38(3.0)$ & $50(3.9)$ \\
\hline $\begin{array}{l}\text { Any AE leading } \\
\text { to discontinu- } \\
\text { ation }\end{array}$ & $24(0.7)$ & $38(1.1)$ & $62(0.9)$ & $10(0.8)$ & $37(2.9)$ \\
\hline
\end{tabular}

All data are $n(\%)$

$A E$ adverse event, bid twice daily, $S A E$ serious adverse event

Table 3 Treatment-emergent adverse events reported in $\geq 2 \%$ of patients (safety analysis set)

\begin{tabular}{|c|c|c|c|c|}
\hline \multirow[t]{2}{*}{ MedDRA preferred term } & \multicolumn{2}{|c|}{ SYGMA 1 + SYGMA 2 pooled safety population } & \multicolumn{2}{|l|}{ SYGMA 1} \\
\hline & $\begin{array}{l}\text { As-needed budesonide- } \\
\text { formoterol } 200 / 6 \mu \mathrm{g} \\
(n=3366)\end{array}$ & $\begin{array}{l}\text { Budesonide maintenance } 200 \\
\mu \mathrm{g} \text { bid }(n=3369)\end{array}$ & $\begin{array}{l}\text { As-needed budesonide- } \\
\text { formoterol } \\
200 / 6 \mu \mathrm{g}(n=1277)\end{array}$ & $\begin{array}{l}\text { As-needed terbuta- } \\
\text { line } 0.5 \mathrm{mg}(n= \\
1277)\end{array}$ \\
\hline Viral URTI & $230(6.8)$ & $252(7.5)$ & $75(5.9)$ & $79(6.2)$ \\
\hline URTI & $152(4.5)$ & $182(5.4)$ & $71(5.6)$ & $76(6.0)$ \\
\hline Asthma $^{\mathrm{a}}$ & $133(4.0)$ & $154(4.6)$ & $37(2.9)$ & $109(8.5)$ \\
\hline Pharyngitis & $84(2.5)$ & $111(3.3)$ & $33(2.6)$ & $34(2.7)$ \\
\hline Bronchitis & $97(2.9)$ & $115(3.4)$ & $33(2.6)$ & $41(3.2)$ \\
\hline Headache & $75(2.2)$ & $80(2.4)$ & $23(1.8)$ & $25(2.0)$ \\
\hline Rhinitis allergic & $79(2.3)$ & $63(1.9)$ & $28(2.2)$ & $28(2.2)$ \\
\hline
\end{tabular}

All data are presented as $n(\%)$

$A E$ adverse event, bid twice daily, $D A E$ discontinuation due to AE, MedDRA Medical Dictionary for Regulatory Activities, $S A E$ serious AE, $U R T I$ upper respiratory tract infection

${ }^{a}$ Asthma symptoms were recorded as an AE only when they met SAE or DAE criteria or the event was new to the patient or inconsistent with the pre-existing asthma history, as judged by the investigator 
not show any differences between patient subpopulations according to sex or age group (data not shown).

\subsection{Discontinuations Due to Adverse Events}

In SYGMA 1, more patients in the As-needed terbutaline group than the As-needed budesonide-formoterol group had a DAE (37 [2.9\%] vs. 10 [0.8\%] patients) or a studydefined asthma-related discontinuation $(21$ [1.6\%] vs. 4 [0.3\%] patients) [7]. Asthma was the most common DAE in SYGMA 1.

In the pooled safety population, the proportion of patients with a DAE was similar in the As-needed budesonide-formoterol (24 patients [0.7\%]) and the budesonide maintenance (38 patients [1.1\%]) groups. Asthma-related discontinuations were the most frequent discontinuations, reported in seven patients $(0.2 \%)$ in the As-needed budesonide-formoterol group and in 13 patients $(0.4 \%)$ in the budesonide maintenance group.

\subsection{Serious Adverse Events and Deaths}

A similar proportion of patients had at least one SAE in the As-needed budesonide-formoterol and the As-needed terbutaline groups (38 [3.0\%] vs. 50 [3.9\%] patients) in SYGMA 1 [7]. Asthma was the most frequent SAE in both of these treatment groups, occurring in six patients $(0.5 \%)$ in the Asneeded budesonide-formoterol recipients and in 16 patients $(1.3 \%)$ in the As-needed terbutaline group. In general, there were no notable differences in the nature or incidence of other individual SAEs between As-needed budesonide-formoterol and As-needed terbutaline. The incidence of SAEs was similar between the As-needed budesonide-formoterol and budesonide maintenance groups (104 [3.1\%] vs. 110 [3.3\%] patients) in the pooled safety population. Generally, there were no clinically relevant differences between treatment groups in the nature or incidence of individual SAEs. The most frequent SAE was asthma, reported in 23 patients $(0.7 \%)$ in the As-needed budesonide-formoterol group and in 25 patients $(0.7 \%)$ in the budesonide maintenance group (pooled safety population).

There were two patient deaths in SYGMA 1 (both in the budesonide maintenance group) [7] and two deaths in SYGMA 2 (one each in the As-needed budesonide-formoterol and budesonide maintenance groups) [5]. None of these deaths in the pooled safety population was considered treatment related. One patient death, in the budesonide maintenance group in SYGMA 2, was considered asthma related (acute asthma exacerbation), as described in detail in the original study publication [5].

\subsection{Safety in Patients with High Levels of As-needed Use}

Use of As-needed medication exceeding 8 or 12 inhalations/ day on at least 1 day during the study was uncommon in the As-needed budesonide-formoterol group in both SYGMA 1 and SYGMA 2 and less frequent than in the As-needed terbutaline or budesonide maintenance groups (Fig. 1a and b) $[5,7]$. Less than $1 \%$ of patients in any treatment group had one or more periods of sustained high As-needed use, defined as $>8$ or $>12$ inhalations/day for periods of either $\geq 7$ or $\geq 14$ consecutive days [5, 7].

In the pooled safety population, fewer patients were using more than eight As-needed inhalations/day for periods of $\geq$ 7 consecutive days in the As-needed budesonide-formoterol group $(n=1)$ than in the budesonide maintenance group $(n$ =19). Among these patients, AEs were reported in 10 of the 19 patients in the budesonide maintenance group; none were in the single patient treated with As-needed budesonide-formoterol (Table 4).

\subsection{Potential $\beta_{2}$-Agonist Class Effects}

Overall, there was a very low incidence of potential $\beta_{2^{-}}$ agonist class effects. In each treatment group, the most commonly reported AEs considered $\beta_{2}$-agonist related included headache and cardiac events such as hypertension, increased blood pressure, tachycardia, palpitations, and angina pectoris (Table 5). There were no notable differences in the incidence of these AEs between As-needed terbutaline and As-needed budesonide-formoterol in SYGMA 1 or between As-needed budesonide-formoterol and budesonide maintenance in the pooled safety population (Table 5).

\subsection{Potential Corticosteroid Class Effects}

The proportions of patients with AEs considered a corticosteroid class effect were low and were similar between Asneeded budesonide-formoterol and As-needed terbutaline groups (SYGMA 1) and between As-needed budesonideformoterol and budesonide maintenance groups (pooled safety analysis; 47 [1.4\%] vs. 57 [1.7\%] patients) (Table 6).

Local corticosteroid-related AEs were reported in less than $1 \%$ of patients but were slightly more common in the budesonide maintenance group than in the As-needed budesonide-formoterol group in the pooled analysis. Dysphonia was reported in 17 patients receiving budesonide maintenance $(0.5 \%)$ and eight of those receiving budesonide-formoterol $(0.2 \%)$, and candidiasis was reported in ten patients receiving budesonide maintenance $(0.3 \%)$ and four of those receiving budesonide-formoterol $(0.1 \%)$. Potential systemic corticosteroid effects were infrequent and occurred in a similar proportion of patients in the As-needed 
Fig. 1 Proportion of patients with high As-needed use of budesonide-formoterol or terbutaline (in the As-needed terbutaline group or budesonide maintenance group) on at least 1 day during the study in a SYGMA 1 [7] and b SYGMA 2 [5]. Data are from the full analysis sets in both studies. The number of inhalations in a day is the sum of day- and night-time As-needed inhalations
(A)

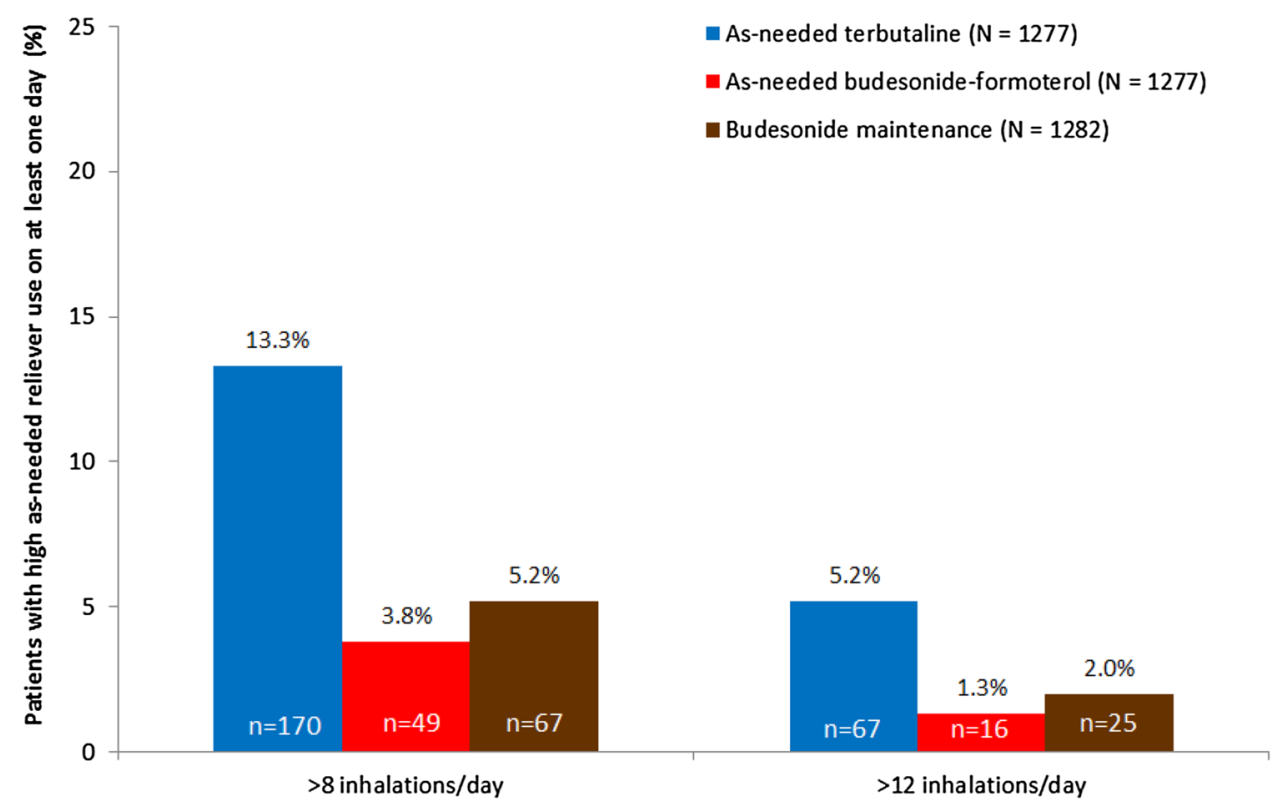

(B)

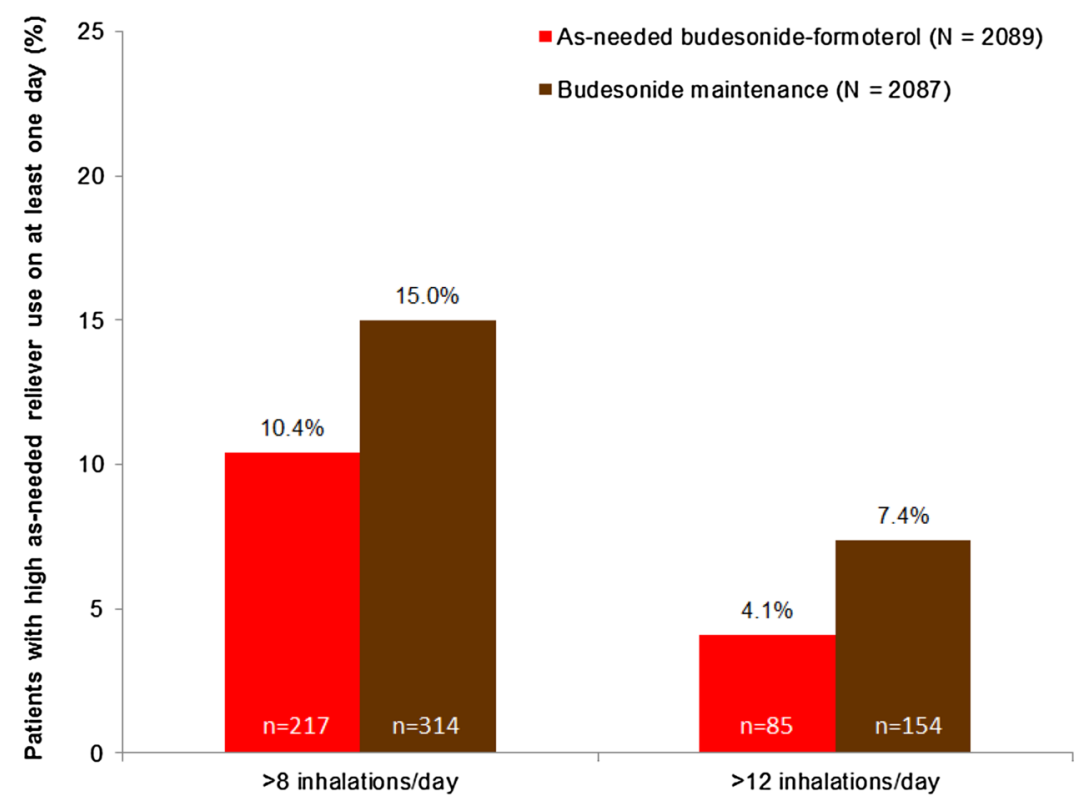

budesonide-formoterol and budesonide maintenance groups (35 [1.0\%] vs. 31 [0.9\%] patients); see Table 6 for further detail. Approximately one-third of the patients reporting potential systemic corticosteroid effects received systemic steroids during the studies. Systemic corticosteroids were adjudged as not likely to have contributed in all but one of the cases, where contribution of the systemic steroids could not be excluded.

Corticosteroid exposure was recorded separately for ICS versus systemic administration. In SYGMA 1 and SYGMA 2 , the median daily dose of ICS was 83 and $75 \%$ lower in the As-needed budesonide-formoterol group than in the budesonide maintenance group, respectively [5, 7]. When ICS exposure from both randomized treatment and the open-label treatment of moderate exacerbations in SYGMA 1 were included in this calculation, the distinction between ICS-containing treatment groups remained: the median total daily ICS dose with As-needed budesonide-formoterol was again $83 \%$ lower than that of budesonide maintenance treatment in SYGMA 1 (60.2 [range 53.7-67.0] vs. $345.2 \mu \mathrm{g}$ [338.0-349.6]) [7] and 75\% lower in SYGMA 2 (65.9 [60.9-72.5] vs. $267.6 \mu \mathrm{g}$ [256.9-273.1]) [5].

In the pooled population, the proportion of patients who had at least one systemic corticosteroid prescription because 
Table 4 All adverse events ${ }^{\mathrm{a}}$ reported for patients with $\geq 7$ consecutive days of more than eight 'as needed' inhalations $(n=20)$, pooled SYGMA 1 and SYGMA 2 populations

\begin{tabular}{|c|c|c|}
\hline Patient & AEs per patient & Treatment group \\
\hline 1 & Viral infection & Budesonide maintenance \\
\hline 2 & Bacterial pneumonia, asthma & Budesonide maintenance \\
\hline 3 & Influenza & Budesonide maintenance \\
\hline 4 & Cataract, viral URTI, asthma & Budesonide maintenance \\
\hline 5 & Viral RTI & Budesonide maintenance \\
\hline 6 & Influenza $^{\mathrm{b}}$ & Budesonide maintenance \\
\hline 7 & Headache & Budesonide maintenance \\
\hline 8 & Headache & Budesonide maintenance \\
\hline 9 & Dyspnoea & Budesonide maintenance \\
\hline 10 & Asthma ${ }^{b}$ & Budesonide maintenance \\
\hline
\end{tabular}

AEs are preferred terms from Medical Dictionary for Regulatory Activities version 20.0. No patients in any of the other randomized treatment groups with an episode of $\geq 7$ consecutive days of more than eight As-needed inhalations/day experienced an $\mathrm{AE}$

$A E$ adverse event, $R T I$ respiratory tract infection, URTI upper RTI

${ }^{a}$ Reports of AEs were collected at study visits so did not necessarily coincide with episodes of sustained high As-needed use

${ }^{\mathrm{b}}$ These AEs were reported on two separate occasions

of asthma was similar between the As-needed budesonideformoterol and budesonide maintenance groups (7.4\% [250 patients] vs. $7.8 \%$ [264 patients]). In SYGMA 1, this proportion was higher in the As-needed terbutaline group than in the As-needed budesonide-formoterol group (12.2\% [156 patients] vs. 5.6\% [71 patients]).

The duration of use of systemic corticosteroids was broadly similar between the As-needed budesonide-formoterol and budesonide maintenance groups in the pooled analysis, with 1818 and 1750 total days of use, respectively. Of those who received any systemic corticosteroids, the median total duration of systemic corticosteroid use was 6 (interquartile range [IQR] 5-9) days per patient in the As-needed budesonide-formoterol group and 6 (IQR 4-8) days per patient in the budesonide maintenance group, with respective ranges of 1-40 and 1-23 days per patient. In SYGMA 1, the total days of systemic corticosteroid use in the As-needed terbutaline group was more than double that in the As-needed budesonide formoterol group (1237 vs. 465 days), although the median duration of use was 6 days per patient in both groups (IQR 4-10 and range 1-51 vs. IQR $5-7$ and range $2-18$, respectively).

\section{Discussion}

Budesonide-formoterol anti-inflammatory reliever therapy is generally well-tolerated in patients with mild asthma. No new safety concerns were identified in this analysis of data from the SYGMA 1 and SYGMA 2 studies in 8012 adult and adolescent patients $[5,7]$, with no safety differences from the well-established safety profile described in the current Symbicort ${ }^{\circledR}$ summary of product characteristics

Table 5 Treatment-emergent AEs representing potential $\beta_{2}$-agonist effects occurring in $\geq 0.1 \%$ of patients (safety analysis set)

\begin{tabular}{|c|c|c|c|c|}
\hline \multirow[t]{2}{*}{ MedDRA preferred term } & \multicolumn{2}{|c|}{ SYGMA $1+$ SYGMA 2 pooled safety population } & \multicolumn{2}{|l|}{ SYGMA 1} \\
\hline & $\begin{array}{l}\text { As-needed budesonide-formo- } \\
\text { terol 200/6 } \mu \mathrm{g} \\
(n=3366)\end{array}$ & $\begin{array}{l}\text { Budesonide maintenance } \\
200 \mu \mathrm{g} \text { bid } \\
(n=3369)\end{array}$ & $\begin{array}{l}\text { As-needed budesonide-formo- } \\
\text { terol } 200 / 6 \mu \mathrm{g} \\
(n=1277)\end{array}$ & $\begin{array}{l}\text { As-needed } \\
\text { terbutaline } \\
0.5 \mathrm{mg} \\
(n=1277)\end{array}$ \\
\hline Any AE & $153(4.5)$ & $145(4.3)$ & $50(3.9)$ & $50(3.9)$ \\
\hline Headache & $75(2.2)$ & $80(2.4)$ & $23(1.8)$ & $25(2.0)$ \\
\hline Hypertension & $41(1.2)$ & $28(0.8)$ & $15(1.2)$ & $13(1.0)$ \\
\hline BP increased & $9(0.3)$ & $9(0.3)$ & $2(0.2)$ & $3(0.2)$ \\
\hline Anxiety & $9(0.3)$ & $3(<0.1)$ & $5(0.4)$ & $1(<0.1)$ \\
\hline Tachycardia & $7(0.2)$ & $8(0.2)$ & $1(<0.1)$ & $3(0.2)$ \\
\hline Angina pectoris & $6(0.2)$ & $3(<0.1)$ & $3(0.2)$ & 0 \\
\hline Tremor & $6(0.2)$ & $7(0.2)$ & $4(0.3)$ & $2(0.2)$ \\
\hline Palpitations & $4(0.1)$ & $7(0.2)$ & $1(<0.1)$ & $1(<0.1)$ \\
\hline Hypokalemia $^{\mathrm{a}}$ & $2(<0.1)$ & 0 & $1(<0.1)$ & $2(0.2)$ \\
\hline Muscle spasms & $4(0.1)$ & $2(<0.1)$ & 0 & 0 \\
\hline Insomnia & $5(0.1)$ & $3(<0.1)$ & $1(<0.1)$ & 0 \\
\hline
\end{tabular}

All data are presented as $n(\%)$

$A E$ adverse event, bid twice daily, BP blood pressure, MedDRA Medical Dictionary for Regulatory Activities

${ }^{\text {a }}$ There was no specific definition of hypokalemia; it was reported as judged by the investigator 
Table 6 Treatment-emergent AEs representing potential corticosteroid effects occurring in at least one patient in any group (safety analysis set)

\begin{tabular}{|c|c|c|c|c|}
\hline \multirow[t]{2}{*}{ Treatment-emergent AEs } & \multicolumn{2}{|c|}{ SYGMA $1+$ SYGMA 2 pooled safety population } & \multicolumn{2}{|l|}{ SYGMA 1} \\
\hline & $\begin{array}{l}\text { As-needed budesonide- } \\
\text { formoterol } 200 / 6 \mu \mathrm{g} \\
(n=3366)\end{array}$ & $\begin{array}{l}\text { Budesonide mainte- } \\
\text { nance } 200 \mu \mathrm{g} \text { bid } \\
(n=3369)\end{array}$ & $\begin{array}{l}\text { As-needed budesonide- } \\
\text { formoterol } 200 / 6 \mu \mathrm{g} \\
(n=1277)\end{array}$ & $\begin{array}{l}\text { As-needed } \\
\text { terbutaline } \\
0.5 \mathrm{mg} \\
(n=1277)\end{array}$ \\
\hline \multicolumn{5}{|l|}{ Potential local steroid effects } \\
\hline Candidiasis & $4(0.1)$ & $10(0.3)$ & 0 & $2(0.2)$ \\
\hline Candida infection & $1(<0.1)$ & 0 & 0 & $1(<0.1)$ \\
\hline Oral candidiasis & $3(<0.1)$ & $4(0.1)$ & - & - \\
\hline Oropharyngeal candidiasis & 0 & $6(0.2)$ & 0 & $1(<0.1)$ \\
\hline Dysphonia & $8(0.2)$ & $17(0.5)$ & $1(<0.1)$ & 0 \\
\hline \multicolumn{5}{|l|}{ Potential systemic steroid effects } \\
\hline Adrenal insufficiency & 0 & $1(<0.1)$ & 0 & 0 \\
\hline DM/DM inadequately controlled & $1(<0.1)$ & $6(0.2)$ & $1(<0.1)$ & $1(<0.1)$ \\
\hline Metabolic bone effects and fractures & $14(0.4)$ & $8(0.2)$ & $6(0.5)$ & $4(0.3)$ \\
\hline Cataract or glaucoma & $4(0.1)$ & $3(<0.1)$ & $1(<0.1)$ & $1(<0.1)$ \\
\hline Psychiatric disorders & $7(0.2)$ & $6(0.2)$ & $1(<0.1)$ & $1(<0.1)$ \\
\hline Contusion & $9(0.3)$ & $5(0.1)$ & $3(0.2)$ & $4(0.3)$ \\
\hline Dysgeusia & $1(<0.1)$ & $2(<0.1)$ & - & - \\
\hline
\end{tabular}

All data are $n(\%)$. The MedDRA preferred term 'contusion' includes bruising

$A E$ adverse event, bid twice daily, $D M$ diabetes mellitus

(SmPC) [14]. The most common AEs reported were viral URTI, URTI, asthma, pharyngitis, bronchitis, headache, and allergic rhinitis.

The safety profile of As-needed budesonide-formoterol was more favorable than that of As-needed terbutaline, with fewer patients in the As-needed budesonide-formoterol group reporting AEs or DAEs. These results are consistent with the AE rates reported for As-needed albuterol (salbutamol) and budesonide-formoterol anti-inflammatory reliever therapy in the open-label Novel START study in patients with mild asthma $(n=668)$ [16]. In the present analysis, Asneeded budesonide-formoterol had a safety profile similar to that for budesonide maintenance therapy plus As-needed terbutaline in the pooled safety population, with similar proportions of patients reporting AEs, SAEs, and DAEs. Beasley et al. [16] found a lower AE rate with As-needed budesonide-formoterol than with budesonide maintenance treatment in patients with mild asthma. Hardy et al. [17] also reported generally similar AE rates between these two treatments in patients with mild to moderate asthma in the PRACTICAL study. Results of the current analysis are broadly consistent with those of other studies that included a treatment group of budesonide maintenance in terms of $\mathrm{AE}$ or SAE rates; in these studies, the comparators were budesonide-formoterol MART $[9,11]$ and/or budesonideformoterol as maintenance (plus As-needed SABA) [8, 9, 11]. Further evidence supporting the safety profile of the MART regimen has been shown in various studies, where safety was similar to that of maintenance budesonide-formoterol plus As-needed SABA [4, 6, 10].

In our safety analysis, there were no treatment-related deaths, and the overall SAE rate was approximately 3-4\%. One of the patient deaths in the SYGMA studies was asthma related and involved a patient on regular inhaled budesonide. The risk of asthma as an SAE or study-defined asthmarelated discontinuations with As-needed budesonide-formoterol was similar to that with budesonide maintenance $(0.5$ vs. $0.7 \%$, and 0.3 vs. $0.4 \%$, respectively), and lower than with As-needed terbutaline (1.3 and $1.6 \%$, respectively).

The SmPC for Symbicort 200/6 $\mu \mathrm{g}$ for adults and adolescents advises a maximum total daily dose of up to 12 inhalations for a limited period of time as part of the approved MART regimen [14]. Overall, few patients used high doses of As-needed therapy in the two SYGMA studies, and an even smaller fraction $(<1 \%)$ used As-needed therapy in a sustained manner ( $\geq 7$ consecutive days) at least once during the study. In SYGMA 1, fewer patients used high Asneeded doses ( $>8$ or $>12$ As-needed inhalations/day on at least 1 day) in the budesonide-formoterol group compared with the As-needed terbutaline group (3.8 vs. $13.3 \%$, and 1.3 vs. $5.2 \%$, respectively). Among those with any episodes of sustained high use (more than eight As-needed inhalations/ day for $\geq 7$ consecutive days), AEs were more common in the budesonide maintenance group (in which the As-needed inhaler was terbutaline) than in the As-needed budesonideformoterol group in the pooled safety population. These data 
further support the safe use of the anti-inflammatory reliever approach to mild asthma treatment using low-dose budesonide-formoterol and should provide added reassurance to clinicians concerned about the safety of this approach in the management of mild asthma.

Our analysis did not find any clinically notable differences in $\beta_{2}$-agonist class-related AEs between As-needed budesonide-formoterol and As-needed terbutaline in SYGMA 1. Budesonide maintenance recipients in SYGMA 1 and 2 also used terbutaline as needed, and, in the pooled safety population from these studies, the $\beta_{2}$-agonist classrelated AE profile was similar. Thus, formoterol as a reliever does not appear to pose any additional safety concerns when taken in combination with budesonide. This conclusion is supported by the similar lack of notable differences in $\beta_{2^{-}}$ agonist class-related AEs between budesonide-formoterol given as MART versus budesonide maintenance $[9,11]$ or budesonide-formoterol as maintenance therapy $[6,9,10]$ in prior studies.

Large clinical safety studies of budesonide-formoterol and other approved ICS-LABA combinations given as maintenance therapy, initiated by the US FDA [26], have dispelled any lingering safety concerns (or misperceptions) relating to serious asthma-related AEs with LABA treatment when taken with an ICS from the same device [27-30]. The FDA subsequently removed the boxed warning from all approved ICS-LABA combinations [31]. As-needed LABA use along with ICS did not reveal any additional concerns.

Dose-related local effects of ICS are well-known [32]. Patients in the SYGMA studies were instructed to rinse their mouth after morning and evening doses of maintenance treatment (budesonide or placebo) but not after doses from the As-needed inhaler (terbutaline or budesonide-formoterol). This instruction is the same as has been previously included in the protocols of a number of other studies with budesonide-formoterol as MART $[6,9,10,33]$. In our analysis, the proportion of patients experiencing local ICS AEs was very low overall, with only slight differences between those using As-needed budesonide-formoterol and those on budesonide maintenance therapy. Local candidiasis (candida infection, oral candidiasis, oropharyngeal candidiasis) was reported in $0.1 \%$ of the As-needed budesonide-formoterol group and in $0.3 \%$ of the budesonide maintenance group (pooled safety population). This supports the clinical advice that there is no need for mouth rinsing when patients use their budesonide-formoterol inhaler as needed, compared with budesonide maintenance.

The broadly similar duration of systemic corticosteroid treatment between both ICS-containing treatment groups in SYGMA 1 and SYGMA 2 was reflected in a similar incidence of potentially corticosteroid-related systemic effects.

A limitation of this analysis includes the need for caution when interpreting the AE data for high As-needed users, since AEs were reported during clinical visits rather than on the day of occurrence, meaning AEs coinciding with the days of high As-needed use are susceptible to recall bias. Further limitations from a safety point of view are the 1-year duration of the SYGMA studies, which did not allow for collection of longer-term safety data, and the observational nature of the collection of some AEs.

\section{Conclusion}

Analysis of safety results from SYGMA 1 and SYGMA 2 confirms that As-needed budesonide-formoterol was welltolerated in the mild asthma population, with no new safety concerns. The safety profile of As-needed budesonideformoterol was favorable compared with that of As-needed terbutaline and similar to that of daily budesonide plus Asneeded terbutaline and offers the advantages of being associated with a very low rate of patients requiring high use of their anti-inflammatory reliever therapy and a lower total exposure to corticosteroids.

Supplementary Information The online version contains supplementary material available at https://doi.org/10.1007/s40264-020-01041-z.

Acknowledgements This pooled analysis was supported by AstraZeneca. The authors thank Audrone Aksomaityte of Phastar, UK, for statistical support and preparation of the initial tables, which formed the basis of the data presented. The authors also thank Tracy Harrison and Stefan Courtney of inScience Communications, Springer Healthcare Ltd, UK, for providing medical writing support, which was funded by AstraZeneca in accordance with Good Publication Practice (GPP3) guidelines (http://www.ismpp.org/gpp3).

\section{Declarations}

Funding This analysis was funded by AstraZeneca.

Conflicts of interest Paul M. O'Byrne received grants and personal fees from AstraZeneca during the conduct of the study; grants and personal fees from GlaxoSmithKline; personal fees from Menarini; and grants from MedImmune, AllerGen NCE, Genentech, Novartis, Merck, and Bayer outside of the submitted work. J. Mark FitzGerald received grants and personal fees from AstraZeneca during the conduct of the study; grants paid to his institution from the Canadian Institute for Health Research, the National Institutes for Health, AllerGen NCE, AstraZeneca, Novartis, Boehringer Ingelheim, GlaxoSmithKline, and Sanofi-Aventis outside the submitted work; and personal fees from GlaxoSmithKline, Novartis, Sanofi Regeneron, and Boehringer Ingelheim; and is a Member of the Global Initiative for Asthma (GINA) Science Committee and the GINA Board. Eric D. Bateman has received personal fees from AstraZeneca, ALK, Boehringer Ingelheim, Menarini, Novartis, Orion, Regeneron, and Sanofi-Genzyme and is a member of the GINA Science Committee and the GINA Board. Peter J. Barnes received grants and personal fees from AstraZeneca during the conduct of the study. Jinping Zheng has received personal fees from AstraZeneca. Stefan Ivanov, Rosa Lamarca, Ulrika Larsdotter, Ulrika Emerath, Gerreke Jansen, Margareta Puu, Vijay Alagappan, and Filip Surmont are all employees of AstraZeneca. Helen K. Reddel re- 
ceived personal fees and non-financial support from AstraZeneca during the conduct of the study; grants, personal fees, and non-financial support from AstraZeneca and GlaxoSmithKline; grants and personal fees from Novartis; and personal fees from Sanofi-Genzyme, Merck, Boehringer Ingelheim, Mundipharma, Chiesi, and TEVA outside of the submitted work; and is chair of the GINA Scientific Committee and a member of the GINA board.

Ethics approval and informed consent The SYGMA studies were performed in accordance with the Declaration of Helsinki and Good Clinical Practice. All patients provided written informed consent before participating in the study. The final clinical study protocols, final informed consent forms, and other written information or materials provided to patients were approved by an independent ethics committee.

Data Sharing Data underlying the findings described in this manuscript may be obtained in accordance with AstraZeneca's data sharing policy described at https://astrazenecagrouptrials.pharmacm.com/ST/Submi ssion/Disclosure.

Author Contributions All authors reviewed and edited the manuscript and approved the final version. In addition, J Mark FitzGerald, Paul O’Byrne, Eric Bateman, Peter Barnes, and Helen Reddel contributed to the study concept and design as part of the SYGMA steering committee, and data interpretation. Jinping Zheng, Margareta Puu, Vijay Alagappan, and Filip Surmont contributed to data interpretation. Rosa Lamarca contributed to data preparation, analysis, and interpretation. Stefan Ivanov contributed to study concept and design, and data preparation and interpretation. Ulrika Emerath contributed to study concept and design, and data preparation, analysis and interpretation. Ulrika Larsdotter and Gerreke Jansen contributed to data preparation and analysis and interpretation.

\section{Consent for publication Not applicable.}

Code availability Not applicable.

Open Access This article is licensed under a Creative Commons Attribution-NonCommercial 4.0 International License, which permits any non-commercial use, sharing, adaptation, distribution and reproduction in any medium or format, as long as you give appropriate credit to the original author(s) and the source, provide a link to the Creative Commons licence, and indicate if changes were made. The images or other third party material in this article are included in the article's Creative Commons licence, unless indicated otherwise in a credit line to the material. If material is not included in the article's Creative Commons licence and your intended use is not permitted by statutory regulation or exceeds the permitted use, you will need to obtain permission directly from the copyright holder. To view a copy of this licence, visit http://creativecommons.org/licenses/by-nc/4.0/.

\section{References}

1. Global Initiative for Asthma. 2020 GINA Report, Global Strategy for Asthma Management and Prevention. 2020. www.ginasthma. org/reports. Accessed 16 Apr 2020

2. British Thoracic Society and Scottish Intercollegiate Guidelines Network. SIGN 158 British guideline on the management of asthma. 2019. https://www.brit-thoracic.org.uk/quality-impro vement/guidelines/asthma/. Accessed 16 Apr 2020

3. Sobieraj DM, Weeda ER, Nguyen E, Coleman CI, White CM, Lazarus SC, et al. Association of inhaled corticosteroids and longacting beta-agonists as controller and quick relief therapy with exacerbations and symptom control in persistent asthma: a systematic review and meta-analysis. JAMA. 2018;319(14):1485-96.

4. Atienza T, Aquino T, Fernandez M, Boonsawat W, Kawai M, Kudo T, et al. Budesonide/formoterol maintenance and reliever therapy via turbuhaler versus fixed-dose budesonide/formoterol plus terbutaline in patients with asthma: phase III study results. Respirology. 2013;18(2):354-63.

5. Bateman ED, Reddel HK, O'Byrne P, Barnes PJ, Zhong N, Keen $\mathrm{C}$, et al. As-needed budesonide-formoterol versus maintenance budesonide in mild asthma. N Engl J Med. 2018;378(20):1877-87.

6. Kuna P, Peters MJ, Manjra AI, Jorup C, Naya IP, MartinezJimenez NE, et al. Effect of budesonide/formoterol maintenance and reliever therapy on asthma exacerbations. Int J Clin Pract. 2007;61(5):725-36.

7. O'Byrne P, FitzGerald J, Bateman E, Barnes PJ, Zhong N, Keen $\mathrm{C}$, et al. Inhaled combined budesonide-formoterol as needed in mild asthma. N Engl J Med. 2018;378(20):1865-76.

8. O’Byrne PM, Barnes PJ, Rodriguez-Roisin R, Runnerstrom E, Sandstrom T, Svensson K, et al. Low dose inhaled budesonide and formoterol in mild persistent asthma: the OPTIMA randomized trial. Am J Respir Crit Care Med. 2001;164(8 Pt 1):1392-7.

9. O'Byrne PM, Bisgaard H, Godard PP, Pistolesi M, Palmqvist M, Zhu Y, et al. Budesonide/formoterol combination therapy as both maintenance and reliever medication in asthma. Am J Respir Crit Care Med. 2005;171(2):129-36.

10. Rabe KF, Atienza T, Magyar P, Larsson P, Jorup C, Lalloo UG. Effect of budesonide in combination with formoterol for reliever therapy in asthma exacerbations: a randomised controlled, doubleblind study. Lancet. 2006;368(9537):744-53.

11. Scicchitano R, Aalbers R, Ukena D, Manjra A, Fouquert L, Centanni S, et al. Efficacy and safety of budesonide/formoterol single inhaler therapy versus a higher dose of budesonide in moderate to severe asthma. Curr Med Res Opin. 2004;20(9):1403-18.

12. Corren J, Korenblat PE, Miller CJ, O'Brien CD, Mezzanotte WS. Twelve-week, randomized, placebo-controlled, multicenter study of the efficacy and tolerability of budesonide and formoterol in one metered-dose inhaler compared with budesonide alone and formoterol alone in adolescents and adults with asthma. Clin Ther. 2007;29(5):823-43.

13. Noonan M, Rosenwasser LJ, Martin P, O'Brien CD, O'Dowd L. Efficacy and safety of budesonide and formoterol in one pressurised metered-dose inhaler in adults and adolescents with moderate to severe asthma: a randomised clinical trial. Drugs. 2006;66(17):2235-54.

14. AstraZeneca UK Limited. Symbicort turbohaler 200/6 inhalation powder - summary of product characteristics. Luton: AstraZeneca; 2018.

15. AstraZeneca Pharmaceuticals LP. Symbicort ${ }^{\circledR}$ (budesonide and formoterol fumarate dihydrate) prescribing Information. Wilmington: AstraZeneca; 2017.

16. Beasley R, Holliday M, Reddel HK, Braithwaite I, Ebmeier S, Hancox RJ, et al. Controlled trial of budesonide-formoterol as needed for mild asthma. N Engl J Med. 2019;380(21):2020-30.

17. Hardy J, Baggott C, Fingleton J, Reddel HK, Hancox RJ, Harwood $\mathrm{M}$, et al. Budesonide-formoterol reliever therapy versus maintenance budesonide plus terbutaline reliever therapy in adults with mild to moderate asthma (PRACTICAL): a 52-week, open-label, multicentre, superiority, randomised controlled trial. Lancet. 2019;394(10202):919-28.

18. Reddel HK, FitzGerald JM, Bateman ED, Bacharier LB, Becker A, Brusselle G, et al. GINA 2019: a fundamental change in asthma management: treatment of asthma with short-acting bronchodilators alone is no longer recommended for adults and adolescents. Eur Respir J. 2019;53(6):1901046.

19. Nwaru BI, Ekstrom M, Hasvold P, Wiklund F, Telg G, Janson C. Overuse of short-acting beta2-agonists in asthma is associated 
with increased risk of exacerbation and mortality: a nationwide cohort study of the global SABINA programme. Eur Respir J. 2020;55(4):1901872.

20. O'Connor BJ, Aikman SL, Barnes PJ. Tolerance to the nonbronchodilator effects of inhaled beta 2-agonists in asthma. N Engl J Med. 1992;327(17):1204-8.

21. Price DB, Trudo F, Voorham J, Xu X, Kerkhof M, Jie JLZ, et al. Adverse outcomes from initiation of systemic corticosteroids for asthma: long-term observational study. J Asthma Allergy. 2018;11:193-204.

22. Waljee AK, Rogers MA, Lin P, Singal AG, Stein JD, Marks RM, et al. Short term use of oral corticosteroids and related harms among adults in the United States: population based cohort study. BMJ. 2017;12(357):j1415.

23. Foster JM, Beasley R, Braithwaite I, Harrison T, Holliday M, Pavord I, et al. Patient experiences of As-needed budesonide-formoterol by Turbuhaler ${ }^{\circledR}$ for treatment of mild asthma; a qualitative study. Respir Med. 2020;175:106154.

24. Baggott C, Reddel HK, Hardy J, Sparks J, Holliday M, Corin A, et al. Patient preferences for symptom-driven or regular preventer treatment in mild to moderate asthma: findings from the PRACTICAL study, a randomised clinical trial. Eur Respir J. 2020;55(4):1902073.

25. International Conference on Harmonisation. Clinical safety data management: definitions and standards for expedited reporting E2A 1994. https://www.ich.org/page/efficacy-guidelines. Accessed 14 Dec 2020
26. Spahn JD. Combination inhaled glucocorticoid/long-acting betaagonist safety: the long and winding road. Ann Allergy Asthma Immunol. 2018;121(4):428-33.

27. Peters SP, Bleecker ER, Canonica GW, Park YB, Ramirez R, Hollis $\mathrm{S}$, et al. Serious asthma events with budesonide plus formoterol vs Budesonide Alone. N Engl J Med. 2016;375(9):850-60.

28. Stempel DA, Raphiou IH, Kral KM, Yeakey AM, Emmett AH, Prazma CM, et al. Serious asthma events with fluticasone plus salmeterol versus fluticasone alone. N Engl J Med. 2016;374(19):1822-30.

29. Stempel DA, Szefler SJ, Pedersen S, Zeiger RS, Yeakey AM, Lee LA, et al. Safety of adding salmeterol to fluticasone propionate in children with asthma. N Engl J Med. 2016;375(9):840-9.

30. Weinstein CLJ, Ryan N, Shekar T, Gates D, Lane SJ, Agache I, et al. Serious asthma events with mometasone furoate plus formoterol compared with mometasone furoate. J Allergy Clin Immunol. 2019;143(4):1395-402.

31. Seymour SM, Lim R, Xia C, Andraca-Carrera E, Chowdhury BA. Inhaled corticosteroids and LABAs - removal of the FDA's boxed warning. N Engl J Med. 2018;378(26):2461-3.

32. Rachelefsky GS, Liao Y, Faruqi R. Impact of inhaled corticosteroid-induced oropharyngeal adverse events: results from a metaanalysis. Ann Allergy Asthma Immunol. 2007;98(3):225-38.

33. Bousquet J, Boulet LP, Peters MJ, Magnussen H, Quiralte J, Martinez-Aguilar NE, et al. Budesonide/formoterol for maintenance and relief in uncontrolled asthma vs. high-dose salmeterol/ fluticasone. Respir Med. 2007;101(12):2437-46.

\section{Authors and Affiliations}

\section{J. Mark FitzGerald ${ }^{1}$ - Paul M. O'Byrne ${ }^{2}$ Eric D. Bateman ${ }^{3} \cdot$ Peter J. Barnes ${ }^{4} \cdot$ Jinping Zheng $^{5} \cdot$ Stefan Ivanov $^{6}$. Rosa Lamarca ${ }^{7}$. Ulrika Larsdotter ${ }^{6}$. Ulrika Emerath ${ }^{6} \cdot$ Gerreke Jansen $^{6} \cdot$ Margareta Puu $^{6} \cdot$ Vijay K. T. Alagappan $^{8}$. Filip Surmont $^{9} \cdot$ Helen K. Reddel ${ }^{10}$}

1 Centre for Lung Health, Vancouver Coastal Health Research Institute, University of British Columbia, 2775 Laurel Street, Vancouver, BC V5Z1M9, Canada

2 Firestone Institute for Respiratory Health, St Joseph's Healthcare and Department of Medicine, Michael G. DeGroote School of Medicine, McMaster University, Hamilton, ON, Canada

3 Division of Pulmonology, Department of Medicine, University of Cape Town, Cape Town, South Africa

4 Airway Disease Section, National Heart and Lung Institute, Imperial College, London, UK
$5 \quad$ State Key Laboratory of Respiratory Diseases, National Clinical Research Centre for Respiratory Disease, First Affiliated Hospital, Guangzhou Medical University, Guangzhou, China

6 AstraZeneca, Gothenburg, Sweden

AstraZeneca, Barcelona, Spain

8 AstraZeneca, Gaithersburg, MD, USA

9 AstraZeneca, Cambridge, UK

10 Woolcock Institute of Medical Research, University of Sydney, Sydney, NSW, Australia 